\title{
Improving Query Expansion Using WordNet
}

\author{
Dipasree Pal ${ }^{* 1}$, Mandar Mitra ${ }^{\dagger 1}$, and Kalyankumar Datta ${ }^{\ddagger 2}$ \\ ${ }^{1}$ Indian Statistical Institute, 203 B.T. Road, Kolkata-700108, India \\ ${ }^{2}$ Jadavpur University, 188, Raja SCM Road, Kolkata-700032, India
}

\begin{abstract}
This study proposes a new way of using WordNet for Query Expansion (QE). We choose candidate expansion terms, as usual, from a set of pseudo relevant documents; however, the usefulness of these terms is measured based on their definitions provided in a hand-crafted lexical resource like WordNet. Experiments with a number of standard TREC collections show that this method outperforms existing WordNet based methods. It also compares favorably with established QE methods such as KLD and RM3. Leveraging earlier work in which a combination of QE methods was found to outperform each individual method (as well as other well-known QE methods), we next propose a combinationbased QE method that takes into account three different aspects of a candidate expansion term's usefulness: (i) its distribution in the pseudo relevant documents and in the target corpus, (ii) its statistical association with query terms, and (iii) its semantic relation with the query, as determined by the overlap between the WordNet definitions of the term and query terms. This combination of diverse sources of information appears to work well on a number of test collections, viz., TREC123, TREC5, TREC678, TREC robust new and TREC910 collections, and yields significant improvements over competing methods on most of these collections.
\end{abstract}

Keywords: Query Expansion, Term Distribution, Term Association, Lexical Resources, Candidate Expansion Term, Pseudo Relevant Documents.

\section{Introduction}

Query Expansion (QE) is a widely used technique that attempts to increase the likelihood of a match between the query and relevant documents by adding semantically related terms (called expansion terms) to a user's query. The expanded query is supposed to retrieve more relevant documents, thereby improving overall performance.

\footnotetext{
*dipasree.pal.gmail.com; Corresponding author. Fax.: +91 332577 3035; Tel.: +91 3325752858.

†mandar.mitra@gmail.com; Fax.: +91 332577 3035; Tel.: +91 3325752858.

¥kalyankumardatta@gmail.com; Fax.: +91 332577 3035; Tel.: +91 3324146129
} 
The source of the expansion terms is an important issue in query expansion. Researchers have explored a variety of sources for collecting these terms. Expansion terms may be taken from the whole target collection, or from a few documents retrieved at top ranks in response to the original query. Recently, researchers have explored the idea of collecting expansion terms from the Web (Benderskv, Metzler, \& Croft, 2012), Wikipedia (Li, Luk. Ho, \& Chung, 2007), query logs (Cui. Wen. Nie. \& Ma, 2002) of search engines, etc. Once a set of candidate terms is determined, the aforesaid resources may also be used to determine the importance of these terms. Finally, a few of the candidate expansion terms (CETs) are selected for inclusion in the expanded query.

Lexical resources like Ontology (Bhogal, Macfarlane, \& Smith, 2007) / WordNet (http://WordNet.princeton.edu/) are also sometimes used as the source of expansion terms. Lexical resources used for query expansion may be constructed either manually (e.g. WordNet), or automatically (usually based on co-occurrence information). Since automatically constructed thesauri are usually based on corpus statistics, they may contain linguistic flaws. In contrast, resources like WordNet that are handcrafted by experienced lexicographers are expected to contain less noise.

Thus, WordNet promises to be a good source of candidate expansion terms. A number of previous studies have shown, however, that WordNet does not necessarily work as expected. Voorhees (Voorhees, 1993, 1994) reported that queries expanded using WordNet yield very little improvement, and sometimes result in degraded performance, compared to the original, unexpanded queries. Recently, Fang (Fang, 2008) showed that QE using WordNet results in improved performance within the axiomatic framework. This method demonstrates that an appropriate use of WordNet can indeed help in getting useful results via query expansion.

In this study, we first propose a new and effective way of using WordNet for Query Expansion. The two features that distinguish our approach from earlier work on WordNet-based QE are the following.

- As in many traditional QE methods, we select terms from top retrieved documents as candidates, instead of obtaining candidate expansion terms from WordNet itself.

- The weight of a term depends not only on the semantic similarity between the term and all query terms (as determined by WordNet), but also takes into account the term's rareness, as well as the similarity score of the top-retrieved document(s) in which the candidate term was found.

This method outperforms the existing WordNet based methods.

The main contribution of this paper is a robust combination method which determines the usefulness of candidate expansion terms by considering multiple sources of information. In previous work on QE, several different ways of estimating the usefulness of CETs have been proposed. We have recently found that these different approaches may be combined to determine the usefulness of a term more reliably (Pal, Mitra, \& Datta, 2013). In this study, we extend this idea by looking at:

- the distribution of a CET in the (pseudo) relevant documents as compared to its distribution in 
the complete corpus;

- information about the association of a term with the query terms;

- the semantic relation between a term and the query terms as determined by WordNet.

Based on the hypothesis that a combination of three totally different QE approaches may perform better than each individual method, we propose a new method which considers all of the three aforesaid features of a term.

Our experiments confirm that the proposed approach leads to improved performance. We tested our methods on TREC123, TREC5, TREC678, TREC robust (new) and TREC910 collections. Compared to the baseline (original queries), the proposed method consistently yields improvements that are significant for all the above collections. It also compares favorably with other state of the art QE methods, and improves upon the results reported recently by Fang (2008).

The rest of the article is organized as follows. We discuss related work in the next section. The following section describes the proposed methods. The section 'Experimental Setup' provides details about the IR system used for our experiments, the collections used, evaluation metrics, etc. Experimental results are presented and discussed in the subsequent section. Finally, we conclude in the last section.

\section{Related work}

Early work on automatic query expansion (AQE) dates back to the 1960s. Rocchio's relevance feedback method (Salton, 1971) is still used in its original and modified forms for AQE. The availability of the TREC collections, and the widespread success of AQE on these collections stimulated further research in this area. Carpineto and Romano (2012) provide a recent and comprehensive survey of AQE techniques. We focus here on some WordNet based AQE methods. We also discuss some important AQE techniques that are either distribution- or association-based.

\section{Use of WordNet}

WordNet has been used both for QE and to disambiguate the sense of query words (Lesk, 1986; Baneriee \& Pedersen, 2002, 2003). Our main focus here is on the use of WordNet for QE. A number of issues need to be addressed when using WordNet as a source of CETs.

- If a query word occurs in multiple synsets, which synset(s) should be selected?

- Once some synsets have been selected, which words should be added to the query? Should only synonyms contained in these synsets be added? Or should hyponyms / hypernyms / meronyms / holonyms also be considered? 
In early work, Voorhees (1993, 1994) explored these questions. Given a search topic, she added a "list of hand-selected WordNet synsets containing nouns germane to the topic." Unfortunately, no significant improvements were obtained even after manually selecting "useful" synsets and adding them to the query.

Zhang. Deng. and Li (2009) used WordNet for sense disambiguation of query terms, and then added synonyms of query words to expand the query. On the CACM collection, their method yields an improvement of about 7\% in P@10 over the original unexpanded queries. Liu, Liu, Yu, and Meng (2004) also disambiguate query terms and add synonyms, hyponyms, words from the term's WordNet gloss, etc. They tested their method on TREC9, TREC10, and TREC12 robust queries and obtained very good results compared to other results reported for these datasets.

More recently, Fang (2008) reported positive results for WordNet-based query expansion within an axiomatic retrieval framework. In the method described by Fang, the set of candidate expansion terms consists of all words from all the synsets in which query terms occur. A CET is actually selected on the basis of the vocabulary overlap between its glosses and the glosses of query terms.

In almost all the earlier studies mentioned above, CETs are taken from WordNet itself. In contrast, we choose CETs from top ranked documents, and then use WordNet to determine the semantic relatedness between query words and the candidate terms. In particular, if a term's gloss shares many words with the query words' glosses, we hypothesize (as in (Lesk, 1986) and (Banerjee \& Pedersen, 2003), and later (Fang, 2008)) that this term is related to the query, and is therefore a good CET.

\section{Other query expansion approaches}

We now turn to two other QE methods that are used in this study: association based methods and distribution based methods. Both methods use the set of pseudo-relevant documents (PRD) as a source of expansion term.

Association-based QE techniques. Early work on association-based AQE includes "concept-based" QE (Qiu \& Frei, 1993) and phrasefinder (Croft \& Yufeng, 1994). Both methods make use of term cooccurrence information extracted from a corpus. Local context analysis (LCA) (Xu \& Croft, 2000, 1996) is another well-known method that also selects expansion terms based on whether they have a high degree of co-occurrence with all query terms. However, in LCA, co-occurrence information is obtained from a set of top-ranked documents retrieved in response to the original query, rather than the whole target corpus. Relevance-based language models (Lavrenko \& Croft, 2001) constitute another, more recent, co-occurrence based approach. This method is based on the Language Modeling framework. The query and relevant documents are all assumed to be generated from an underlying relevance model. This model is estimated based on (only) the pseudo relevant documents for a particular query. This approach was subsequently refined by Abdul-Jaleel et al. (2004). The refinement, called RM3, incorporates the original query when estimating the relevance model. According to the comparative studies by Lv and Zhai (2009) and Miao. Huang. and Ye (2012), RM3 is the most effective and robust among a number of 
state-of-the-art AQE methods. RM3 is frequently used as a baseline against which several recent QE methods have been compared (Miao et al., 2012; Lv \& Zhai, 2010; Bendersky, Metzler, \& Croft, 2011; Cao, Nie, Gao, \& Robertson, 2008a; Krikon, Kurland, \& Bendersky, 2010).

Distribution-based QE techniques. As early as 1978, Doszkocs (1978) proposed the interactive use of an associative dictionary that was constructed based on a comparative analysis of term distributions. Also well known is Robertson's analysis of term selection for query expansion (S. E. Robertson, 1991). More recently, Carpineto, Mori, Romano, and Bigi (2001) proposed an effective QE method based on information theoretic principles. This method uses the Kullback-Leibler divergence (KLD) between the probability distributions of terms in the relevant (or pseudo-relevant) documents and in the complete corpus.

Amati (2003) proposes a new distribution based method which uses Bose-Einstein statistics. This method also calculates the divergence between the distribution of terms in the pseudo relevant document set and a random distribution.

Combination-based techniques. Efforts have also been made to combine AQE methods in various ways to improve retrieval effectiveness. Carpineto, Romano, and Giannini (2002) combined the scoring functions of a number of methods, all of them distribution-based, to obtain improvements. A different approach is adopted by Bendersky et al. (2012), who use multiple sources for query formulation. In addition to the target corpus, information from 'ClueWeb (http://boston.lti.cs.cmu.edu/clueweb09/) Heading Text', 'ClueWeb Anchor Text' and Wikipedia (Available at: http://download.wikimedia.org/enwiki/) is used. Additionally, Google N-grams (Available from Linguistic Data Consortium catalog), and a query $\log$ (Available as a part of Microsoft 2006 RFP dataset) from MSN are used to determine term weights.

WordNet has also been used as a part of combination-based methods. The work by Liu et al. (2004) (mentioned in 'related work' section ) combines information from WordNet with information about the correlation (or co-occurence) of words with query terms. Similarly, Fang (2008) also tested a combination method that uses WordNet as well as the mutual information of terms. The combination-based method performed better than the individual methods.

Our earlier work on combination-based QE method (Pal et al., 2013) is different in that it combines a distribution-based method with an association-based method (based on our belief that these two classes of methods offer different advantages). Further, rather than simply combining scores, we used one method to refine the set of terms selected by the other (Of course, this can also, strictly speaking, be regarded as a combination where one component is very highly weighted.). This approach is somewhat similar in spirit to a method proposed by Cao, Nie, Gao, and Robertson (2008b), in which terms selected using standard pseudo relevant feedback (PRF) are refined using a classifier that is trained to differentiate between useful and harmful candidate expansion terms. Our earlier work is most strongly related to that of Pérez-Agüera and Araujo (2008), who also combine co-occurrence-based and distribution-based methods. In their work, the combination is relatively straightforward, however: one method is used for term 
selection and the other for weighting. Word co-occurrence is measured using the Tanimoto coefficient. Distributional differences are measured based on KLD or Bose-Einstein statistics. The methods are tested on a relatively small Spanish dataset. We used the well-known LCA (new) and RM3 methods (instead of Tanimoto coefficient) to quantify term association. Also, instead of simply using one method for term selection and the other for weighting, we combine both methods for selection. Finally, we tested our method on a number of large TREC datasets.

In the present study, we extend our earlier work by first proposing a new and effective way of using WordNet for QE, and then combining this WordNet based method with association based and distribution based AQE methods to improve overall performance.

\section{Proposed Methods}

\section{Proposed WordNet Approach (P-WNET)}

During query expansion, the first important decision is the choice of the source of candidate expansion terms. A set of sample relevant documents for a given query would be a good source of CETs. In the absence of true relevance judgments, the $D$ top-ranked documents retrieved in response to the given query may be regarded as a set of pseudo relevant documents (PRDs). Many well-known QE methods (Carpineto et al., 2001; Xu \& Croft, 2000; Amati \& Van Rijsbergen, 2002) have shown that PRDs are a good source of CETs. In contrast, WordNet-based QE typically starts with the synonyms (and possibly holonyms, meronyms, etc.) of the query words as CETs. Instead of confining ourselves to this set, we consider all terms from PRDs as CETs.

Earlier work has shown that two terms tend to be strongly related if their WordNet definitions share many common terms. Thus, if the definition(s) of a term shares words with query word definitions, then the term may be semantically related to the query, even though it may not be a direct synonym of (or otherwise explicitly related via WordNet relations to) query words. For example, consider a candidate term 'spondylitis' for TREC query 604 (Lyme disease, arthritis). WordNet definitions for the CET spondylitis, query term arthritis and the query phrase 'Lyme disease' share the terms 'inflammation' and 'joint'. Thus, spondylitis appears to be strongly related to the query. Similarly, the term 'ill' (whose definition contains 'disease') is found to be related to 'Lyme disease'.

The above idea has been used to quantify the relationship between a CET $t$ and a query word $q_{i}$ as follows. The definitions of $t$ and $q_{i}$ are considered as two sets of words, and the overlap between these two sets is taken as $\operatorname{Rel}\left(t, q_{i}\right)$, the semantic similarity between $t$ and $q_{i}$. The overlap may be measured using either the Jaccard coefficient (Equation 1), as in (Fang, 2008; Baneriee \& Pedersen, 2003), or the Dice coefficient (Equation 2). Here, $c_{x}$ denotes the number of documents in which term $x$ occurs, and 
$c_{x, y}$ is the number of documents in which $x$ and $y$ cooccur.

$$
\begin{gathered}
\operatorname{Re}_{t, q_{i}}=\frac{c_{t, q_{i}}}{c_{t}+c_{q_{i}}-c_{t, q_{i}}} \\
\operatorname{Re}_{t, q_{i}}=\frac{2 * c_{t, q_{i}}}{c_{t}+c_{q_{i}}}
\end{gathered}
$$

Our experimental results show that Dice co-efficient performs somewhat better than Jaccard co-efficient.

Phrases. Phrases usually have a specific meaning that goes beyond the sum of the meaning of the constituent words. We therefore give more priority to phrases in the query than single words when finding definitions from WordNet. Any two consecutive words are considered as a potential phrase, and such pairs are looked up in WordNet first. If the phrase does not have an entry, then its constituent words are looked up separately. Note that we consider phrases only at the time of finding definitions from WordNet; we do not consider phrases anywhere else. Specifically, documents and queries are indexed using single words only. Thus, all CETs are single words. WordNet definitions are also considered to be bags of single words.

After computing the relationship between a CET $t$ and the query words, we consider the importance of $t$ in the collection as a whole, as given by its idf. We use Robertson's idf (S. Robertson, 2004) formula as shown in Equation 3 ( $N$ denotes the total number of documents in the collection, and $N_{t}$ denotes the number of documents in which term $t$ occurs). If the $i d f$ turns out to be negative, we use a very small number (0.0001) instead.

$$
i d f_{t}=\max \left(0.0001, \log _{10} \frac{N-N_{t}+0.5}{N_{t}+0.5}\right)
$$

Next, we factor in the importance of the pseudo relevant documents in which $t$ occurs. This is intended to capture the intuition that terms coming from relevant document are better than the terms coming from non-relevant documents. In a pseudo relevance feedback setting, this translates to the hypothesis that terms coming from top ranked documents are likely to be more useful as CETs. Accordingly, we modify the score of $t$ by the normalized similarity of documents in which $t$ occurs. Equation 4 shows how all the above factors are combined.

$$
s\left(t, q_{i}\right)=\operatorname{Rel}_{t, q_{i}} * i d f_{t} * \sum_{d \in P R D}\left(\frac{\operatorname{Sim}(d, Q)}{\max _{d^{\prime} \in P R D} \operatorname{Sim}\left(d^{\prime}, Q\right)}\right)
$$

Here, $\operatorname{Sim}(d, Q)$ denotes the similarity score of document $d$ with respect to the query $Q$.

The actual score of a CET $t$ is given by $S(t)$ (Equation 5 ). The summand in Equation 5 is a slowly growing and bounded function of $s\left(t, q_{i}\right)$. This function ensures that the weight of a CET lies within a small range of values.

$$
S(t)=\sum_{q_{i} \in Q} \frac{s\left(t, q_{i}\right)}{1+s\left(t, q_{i}\right)}
$$

The $T$ CETs with the highest $S(t)$ scores are selected for inclusion in the expanded query. The weights 
of terms in the final expanded query are obtained by combining the normalized weights of expansion terms (Equation 6) along with the normalized weights of original query terms (Equation 7), as shown in Equation 8.

$$
\begin{gathered}
\text { score }_{\text {exp }}(t)=\frac{S(t)}{\max _{t^{\prime} \in d \in P R D} S\left(t^{\prime}\right)} \\
\text { score }_{\text {orig }}(t)=\frac{1+\log (t f(t, Q))}{1+\max _{t^{\prime} \in Q} \log \left(t f\left(t^{\prime}, Q\right)\right)} \\
\text { score }(t)=\text { score }_{\text {exp }}(t)+\beta * \text { score }_{\text {orig }}(t)
\end{gathered}
$$

Since our focus is on short, title-only queries that typically contain 2-3 query words with each term occurring only once, Equation 7 assigns a weight of 1 to all query terms most of the time. However, since the original query terms are supplied by the user, we regard them as being more important compared to the automatically added expansion terms (as in (Buckley, Singhal, \& Mitra, 1995)). We therefore set $\beta$ to 2 .

\section{Combination Method (KLWNET)}

As explained in first section, our goal is to improve QE by combining three very different methods for estimating the usefulness of a CET: (i) a distribution based method, (ii) an association based method; and (iii) WordNet. In earlier work (Pal et al., 2013), we have already explored four possible combinations of two distribution based $(K L D, B o 1)$ and two association based methods (LCA, RM3): KLDLCA, KLDRM3, Bo1LCA, and Bo1RM3. We select one of these methods (KLDLCA) and combine it with $P$-WNET (mentioned in the previous subsection).

Our combination method is very simple. We included in the expanded query all the terms suggested by the contributing methods. We use Equation 9 to determine the weights of expansion terms.

$$
\operatorname{score}(t)=\alpha * \operatorname{score}_{1}(t)+(1-\alpha) * \operatorname{score}_{2}(t)
$$

where score $_{1}$ and score $_{2}$ are the normalized scores of a term computed using $P$-WNET and KLDLCA, respectively. In this formula, the parameter $\alpha$ is used to control the relative importance of $P$ - WNET and $K L D L C A$. We experimented with values of $\alpha$ ranging from 0.1 to 0.9 in increments of 0.1 on our training corpus (details in the next section), and found $\alpha=0.3$ works well. This is consistent with the fact that, in isolation, KLDLCA performs better than P-WNET (Table 4). Thus, a small value of alpha should be preferred.

\section{Experimental Setup}

Table 1 lists the details of the test collections used in our experiments. Since the TREC678 collection consists of a large number of queries (150), and is more recent compared to the TREC123 collection, it 


\begin{tabular}{ccc}
\hline Query Id. & \# of Queries & Documents \\
\hline TREC123 & 150 & TREC disks 1, 2 \\
51-200 & & \\
\hline TREC5 & 50 & TREC disks 2, 4 \\
251-300 & & \\
\hline TREC678 & 150 & TREC disks 4, 5 - CR \\
301-450 & & \\
\hline ROBnew & 100 & TREC disks 4, 5 - CR \\
$601-700$ & & \\
\hline TREC910 & 100 & WT10G \\
$451-550$ & & \\
\hline
\end{tabular}

Table 1: Test collections

is used as a "training set" for the purpose of tuning the parameters of our methods. As real-life queries are very short, we used only the title field of all queries. Many of the queries thus contain only one term, and most of the remainder are no longer than three words.

We used the TERRIER (http://terrier.org/) retrieval system for our experiments. At the time of indexing, stopwords are removed and Porter's stemmer is used as preprocessing. All documents and queries are indexed using single terms, no phrases are used. The IFB2 variant of the Divergence From Randomness (DFR) model (Amati \& Van Rijsbergen, 2002) - a relatively recent model that performs well across test collections — is used for term-weighting in all our experiments as it performs better compared to the other variants available within TERRIER. Parameters are set to the default values used in TERRIER.

Results are evaluated using standard evaluation metrics (Mean Average Precision (MAP), Geometric Mean Average Precision (GM_MAP), precision at top 10 ranks (P@10), and overall recall (number of relevant documents retrieved)). Additionally, for each expansion method, we report the percentage of queries for which the method resulted in an improvement in MAP of more than 5\% over the baseline (no feedback). This number may be viewed as indicative of how safe or robust an expansion method is. We use a two-tailed paired $t$-test with a confidence level of $95 \%$ to check for statistically significant differences.

\section{Experimental Results}

The objectives of our experiments are two-fold.

- (Experiment 1) To explore the effectiveness of the proposed WordNet based QE method (P-WNET), and to compare it with the baseline as well as existing WordNet based methods. We analyze the results in order to estimate the contribution of each factor in Equation 4 to the overall retrieval effectiveness. We also compare the results with those obtained using two state-of-the-art methods, and show that our proposed approach compares favorably with these methods.

- (Experiment 2) To evaluate KLWNET, which combines distribution based information, association 
based information and information from WordNet. This method is also compared to state-of-the-art QE methods.

\section{Experiment 1: Proposed WordNet Approach (P-WNET)}

We use the following baselines for comparison in Tables 2 and 3. To the best of our knowledge, no comparisons between WordNet-based methods and other state-of-the-art QE methods such as KLD (Carpineto et al.) 2001) and RM3 (Abdul-Jaleel et al., 2004) have been presented in earlier work. However, reported figures for WordNet-based QE techniques are generally much lower than the figures reported for these methods. We include these two methods in our list of baselines.

- No feedback. The original, unexpanded queries are used for retrieval using the baseline method described in the section 'Experimental Setup'.

- FANG. The results reported by Fang in (Fang, 2008) are included in Table 3 under this label.

- FN-PW. This method is very similar to the approach described by Fang (2008), except for the basic setup, which is adopted from P-WNET. The two differences between FN-PW and FANG are (i) in FN-PW, CETs are obtained from the top ranked documents, while Fang selects CETs from the synsets of query words; (ii) FN-PW uses the DFR model for term weighting, while Fang's work is based on the axiomatic framework. The motivation here is to study the effect of these changes on Fang's approach.

- No-WNet. Recall from Equation 4 that P-WNET combines information from WordNet with (i) information about the idf of a term, and (ii) the goodness of the document(s) from which the candidate term is obtained. No-WNet omits the WordNet-based factor in Equation 4 and makes use of only the last two sources of information mentioned above.

- KLD. We choose KLD (Carpineto et al., 2001) as a state-of-the-art representative of distributionbased AQE methods. The parameter settings chosen for this method are as follows.

- $D$, the number of top-ranked documents that are assumed to be relevant, is set to 10;

- $T$, the number of expansion terms is set to 40 .

These settings are in agreement with the observations of Carpineto et al. (2001). Our experiments also confirm that these values work well for KLD across collections.

Note that the results presented here correspond to our implementation of KLD within TERRIER. While our implementation provides better results than TERRIER's native implementation of KLD, we were not able to exactly replicate the results reported in (Carpineto et al., 2001). This is likely due to differences between the retrieval functions, indexing or query processing. For example, using full queries (title, desc and narr) on the TREC8 collection, and BM25 as the base term-weighting 
formula, we get MAP scores of 0.2992 for KLD (compared to a baseline of 0.2625). When using the IFB2 model, however, the baseline is higher (MAP $=0.2753$ ), but KLD appears less effective $(\mathrm{MAP}=0.2850)$.

- RM3. This is also a relatively recent state-of-the-art AQE method. We use $D=50$ documents (as suggested in (Lavrenko \& Croft, 2001)) and $T=50$ terms. We set the Dirichlet smoothing parameter $(\mu)$ to 2500 and the interpolation parameter to 0.5 , based on the default settings for these parameters in Lemur (http://www.lemurproject.org/). As before, we used the TREC678 collection to verify that these parameter values work well for us. In fact, for a number of datasets, our results for RM3 are superior to those reported in other recent papers ((Bendersky et al., 2011), for example).

Parameter settings. For our proposed method $P$-WNET, and for the training set mentioned in the previous section, a choice of $D=10$ documents and $T=60$ terms works well. We used these same parameters for the No-WNet method, as the main motivation behind this method is to test the effectiveness of the Wordnet-based component in P-WNET. We also tested various parameter combinations for the FN-PW method on the TREC678 collection, and found that these same values are appropriate for this method as well. Parameter settings for the other methods (KLD and RM3) are discussed above.

From Table 2, it is clear that the proposed method P-WNET is significantly better than the "No feedback" baseline on all collections. Of course, our main concern is to compare our method with other state-of-the-art QE methods. Our results show that P-WNET performs better than FN-PW - a method that is very similar to Fang's in terms of technique as well as performance - on all collections, with the improvements being significant for the TREC123, TREC678, and ROBnew collections. P-WNET also does better than RM3 on all collections, with the difference being significant for the TREC123 and TREC678 collections. P-WNET is comparable to KLD, with neither method being significantly better than the other.

Table 3 presents a direct comparison between P-WNET and FANG on the two collections used in (Fang, 2008). It shows that our "no feedback" baseline is close to the baseline used by Fang, but P-WNET yields MAP values that are a good deal higher than the figures reported in her paper. To make comparisons easy, MAP values for KLD and RM3 are also included in Table 3. While FANG does slightly better than RM3, KLD yields the best figures; these are noticeably higher than those for FANG, but not significantly better than the figures for P-WNET.

\section{Experiment 2: Combination Method (KLWNET)}

We now turn to our combination based method. We compare KLWNET to KLD, RM3, P-WNET and KLDLCA (Pal et al., 2013), a combination-based QE method that we proposed in earlier work (see the 


\begin{tabular}{|c|c|c|c|c|c|c|c|}
\hline Dataset & Measure & Baseline & FN-PW & no-wnet & KLD & RM3 & P-WNET \\
\hline \multirow[t]{8}{*}{ TREC123 } & MAP & 0.218 & $\begin{array}{c}0.242 \\
(10.8)\end{array}$ & $\begin{array}{l}0.262 \\
(20.0)\end{array}$ & $\begin{array}{l}\mathbf{0 . 2 7 4} \\
(25.4)\end{array}$ & $\begin{array}{l}0.249 \\
(14.1)\end{array}$ & $\begin{array}{c}0.273^{B, p, n, r} \\
(24.9)\end{array}$ \\
\hline & GM_MAP & 0.097 & 0.111 & 0.096 & 0.101 & 0.109 & 0.112 \\
\hline & & & $(14.4)$ & $(-0.8)$ & $(4.8)$ & $(13.1)$ & (15.9) \\
\hline & $\mathrm{P} @ 10$ & 0.481 & 0.515 & 0.525 & 0.537 & 0.511 & 0.526 \\
\hline & & & $(7.2)$ & $(9.3)$ & $(11.8)$ & $(6.2)$ & $(9.4)$ \\
\hline & \#rel_ret & 16536 & 17345 & 17901 & 18299 & 17702 & 18377 \\
\hline & & & $(4.9)$ & $(8.3)$ & (10.7) & $(7.1)$ & (11.1) \\
\hline & $>$ baseline on & 0 & 60 & 58 & 62 & 64 & 68 \\
\hline \multirow[t]{8}{*}{ TREC5 } & MAP & 0.157 & $\begin{array}{c}0.164 \\
(4.3)\end{array}$ & $\begin{array}{l}0.154 \\
(-2.3)\end{array}$ & $\begin{array}{c}0.168 \\
(6.9)\end{array}$ & $\begin{array}{c}\mathbf{0 . 1 7 0} \\
(8.2)\end{array}$ & $\begin{array}{c}\mathbf{0 . 1 7 0}^{n} \\
(8.4)\end{array}$ \\
\hline & GM_MAP & 0.043 & 0.047 & 0.030 & 0.035 & 0.045 & 0.042 \\
\hline & & & $(11.3)$ & $(-28.5)$ & $(-18.4)$ & $(7.1)$ & $(-0.2)$ \\
\hline & $\mathrm{P} @ 10$ & 0.286 & 0.326 & 0.284 & 0.268 & 0.336 & 0.310 \\
\hline & & & $(14.0)$ & $(-0.7)$ & $(-6.3)$ & $(17.5)$ & $(8.4)$ \\
\hline & \#rel_ret & 1936 & 2075 & 1945 & 2184 & 2077 & 2383 \\
\hline & & & $(7.2)$ & $(0.5)$ & (12.8) & $(7.3)$ & $(23.1)$ \\
\hline & $>$ baseline on & 0 & 48 & 32 & 42 & 50 & 44 \\
\hline \multirow[t]{8}{*}{ TREC678 } & MAP & 0.218 & $\begin{array}{l}0.233 \\
(6.6)\end{array}$ & $\begin{array}{l}0.234 \\
(7.5)\end{array}$ & $\begin{array}{l}\mathbf{0 . 2 5 7} \\
(18.0)\end{array}$ & $\begin{array}{l}0.230 \\
(5.6)\end{array}$ & $\begin{array}{c}0.255^{B, p, n, r} \\
(17.0)\end{array}$ \\
\hline & GM_MAP & 0.100 & 0.119 & 0.099 & 0.101 & 0.106 & 0.125 \\
\hline & & & (19.1) & $(-0.5)$ & $(1.3)$ & $(5.9)$ & $(25.5)$ \\
\hline & $\mathrm{P} @ 10$ & 0.431 & 0.448 & 0.429 & 0.438 & 0.435 & 0.451 \\
\hline & & & $(3.9)$ & $(-0.6)$ & $(1.6)$ & $(0.8)$ & $(4.6)$ \\
\hline & \#rel_ret & 7287 & 7638 & 7770 & 8556 & 7617 & 8246 \\
\hline & & & $(4.8)$ & $(6.6)$ & $(17.4)$ & $(4.5)$ & $(13.2)$ \\
\hline & $>$ baseline on & 0 & 54 & 49 & 52 & 45 & 55 \\
\hline \multirow[t]{9}{*}{ ROBnew } & MAP & 0.278 & 0.296 & 0.302 & 0.312 & 0.305 & $0.321^{B, p, n}$ \\
\hline & & & $(6.5)$ & $(8.6)$ & $(12.2)$ & $(9.8)$ & (15.4) \\
\hline & GM_MAP & 0.179 & 0.198 & 0.171 & 0.182 & 0.199 & 0.200 \\
\hline & & & (10.5) & $(-4.6)$ & $(2.0)$ & $(11.2)$ & (11.7) \\
\hline & $\mathrm{P} @ 10$ & 0.421 & 0.432 & 0.437 & 0.405 & 0.442 & 0.437 \\
\hline & & & $(2.6)$ & $(3.8)$ & $(-3.8)$ & $(5.0)$ & $(3.8)$ \\
\hline & \#rel_ret & 2887 & 3050 & 3082 & 3172 & 3002 & 3143 \\
\hline & & & $(5.6)$ & $(6.8)$ & $(9.9)$ & $(4.0)$ & $(8.9)$ \\
\hline & $>$ baseline on & 0 & 52 & 53 & 52 & 56 & 58 \\
\hline \multirow[t]{9}{*}{ TREC910 } & MAP & 0.195 & 0.206 & 0.188 & 0.193 & 0.211 & $0.213^{n}$ \\
\hline & & & $(5.5)$ & $(-4.0)$ & $(-1.1)$ & $(8.0)$ & $(9.0)$ \\
\hline & GM_MAP & 0.081 & 0.089 & 0.055 & 0.056 & 0.087 & 0.081 \\
\hline & & & $(10.0)$ & $(-32.2)$ & $(-30.2)$ & $(7.4)$ & $(0.2)$ \\
\hline & $\mathrm{P} @ 10$ & 0.307 & 0.331 & 0.310 & 0.293 & 0.329 & 0.336 \\
\hline & & & $(7.7)$ & $(1.0)$ & $(-4.6)$ & $(7.0)$ & $(9.3)$ \\
\hline & \#rel_ret & 3770 & 3916 & 3440 & 3987 & 3889 & 3981 \\
\hline & & & $(3.9)$ & $(-8.8)$ & $(5.8)$ & $(3.2)$ & $(5.6)$ \\
\hline & $>$ baseline on & 0 & 55 & 38 & 44 & 53 & 47 \\
\hline
\end{tabular}

Table 2: Improvements obtained using P-WNET on different datasets. The "> baseline on" line shows the \%-age of queries for which each method beats the baseline by $>5 \%$. Superscripts $B, p, n, k, r$ denote a statistically significant improvement of the proposed method over the baseline (no feedback), FN-PW, no-wnet, KLD and RM3 respectively. For a particular collection, the highest value for any metric is shown in bold.

'Related Work' section). In KLDLCA, candidate expansion terms are first obtained (and weighted) using a distribution based method (KLD); these terms are reranked (but not reweighted) based on local context analysis; the top terms from the reranked list are included in the final expanded query.

Parameter settings. For KLDLCA, we set $D=50$ documents and $T=40$ terms, since these values work well on our training dataset. For the KLWNET method, which combines KLDLCA with P-WNET, we use the best parameter settings for each of the constituent methods, i.e., $D=50$ documents and $T=40$ terms for KLDLCA, and $D=10, T=60$ for P-WNET (see 'Experiment 1' section).

Table 4 shows that KLWNET yields the highest MAP among all methods on all the datasets. On

\begin{tabular}{cccccccc}
\hline Dataset & Baseline & $\begin{array}{c}\text { Baseline } \\
\text { (Fang, 2008) }\end{array}$ & FN-PW & KLD & RM3 & P-WNET & $\begin{array}{c}\text { FANG } \\
\text { (Fang, 2008) }\end{array}$ \\
\hline TREC7 & 0.1891 & 0.1860 & 0.2020 & 0.2568 & 0.2025 & 0.2437 & 0.2160 \\
TREC8 & 0.2467 & 0.2500 & 0.2622 & 0.2861 & 0.2591 & 0.2853 & 0.2660 \\
\hline
\end{tabular}

Table 3: Comparison between Fang's WordNet-based method and P-WNET. 


\begin{tabular}{|c|c|c|c|c|c|c|c|}
\hline Dataset & Measure & Baseline & KLD & RM3 & P-WNET & KLDLCA & KLWnet \\
\hline \multirow[t]{8}{*}{ TREC123 } & MAP & 0.218 & $\begin{array}{l}0.274 \\
(25.4)\end{array}$ & $\begin{array}{l}0.249 \\
(141)\end{array}$ & $\begin{array}{l}0.273 \\
(249)\end{array}$ & $\begin{array}{l}0.283 \\
(29.3)\end{array}$ & $\mathbf{0 . 2 9 0} 0^{B, k, r, w, k l}$ \\
\hline & GM_MAP & 0.097 & 0.101 & 0.109 & 0.112 & 0.105 & 0.114 \\
\hline & & & $(4.8)$ & (13.1) & (15.9) & $(8.4)$ & $(17.6)$ \\
\hline & $\mathrm{P} @ 10$ & 0.481 & 0.537 & 0.511 & 0.526 & 0.567 & 0.550 \\
\hline & & & $(11.8)$ & $(6.2)$ & $(9.4)$ & $(17.9)$ & $(14.4)$ \\
\hline & \#rel_ret & 16536 & 18299 & 17702 & 18377 & 18850 & 19138 \\
\hline & & & $(10.7)$ & $(7.1)$ & (11.1) & $(14.0)$ & (15.7) \\
\hline & $>$ baseline on & 0 & 62 & 64 & 68 & 65 & 69 \\
\hline \multirow[t]{8}{*}{ TREC5 } & MAP & 0.157 & $\begin{array}{l}0.168 \\
(6.9)\end{array}$ & $\begin{array}{l}0.170 \\
(8.2)\end{array}$ & $\begin{array}{l}0.170 \\
(8.4)\end{array}$ & $\begin{array}{l}0.171 \\
(9.0)\end{array}$ & $\begin{array}{c}\mathbf{0 . 1 7 7 ^ { B , k , k T }} \\
(12.6)\end{array}$ \\
\hline & GM_MAP & 0.043 & 0.035 & 0.045 & 0.042 & 0.036 & 0.040 \\
\hline & & & $(-18.4)$ & $(7.1)$ & $(-0.2)$ & $(-14.6)$ & $(-5.6)$ \\
\hline & $\mathrm{P} @ 10$ & 0.286 & 0.268 & 0.336 & 0.310 & 0.274 & 0.306 \\
\hline & & & $(-6.3)$ & $(17.5)$ & $(8.4)$ & $(-4.2)$ & $(7.0)$ \\
\hline & \#rel_ret & 1936 & 2184 & 2077 & 2383 & 2218 & 2294 \\
\hline & & & $(12.8)$ & $(7.3)$ & $(23.1)$ & $(14.6)$ & $(18.5)$ \\
\hline & $>$ baseline on & 0 & 42 & 50 & 44 & 52 & 56 \\
\hline \multirow[t]{9}{*}{ TREC678 } & MAP & 0.218 & 0.257 & 0.230 & 0.255 & 0.266 & $0.271^{B, k, r, w, k l}$ \\
\hline & & & $(18.0)$ & $(5.6)$ & $(17.0)$ & $(22.0)$ & $(24.4)$ \\
\hline & GM_MAP & 0.100 & 0.101 & 0.106 & 0.125 & 0.103 & 0.117 \\
\hline & & & $(1.3)$ & $(5.9)$ & $(25.5)$ & $(3.7)$ & $(17.2)$ \\
\hline & $\mathrm{P} @ 10$ & 0.431 & 0.438 & 0.435 & 0.451 & 0.441 & 0.446 \\
\hline & & & $(1.6)$ & $(0.8)$ & $(4.6)$ & $(2.2)$ & $(3.4)$ \\
\hline & \#rel_ret & 7287 & 8556 & 7617 & 8246 & 8567 & 8658 \\
\hline & & & $(17.4)$ & $(4.5)$ & $(13.2)$ & $(17.6)$ & $(18.8)$ \\
\hline & $>$ baseline on & 0 & 52 & 45 & 55 & 57 & 64 \\
\hline \multirow[t]{9}{*}{ ROBnew } & MAP & 0.278 & 0.312 & 0.305 & 0.321 & 0.326 & $\mathbf{0 . 3 3 5 ^ { B , k , r , w , k l }}$ \\
\hline & & & $(12.2)$ & $(9.8)$ & $(15.4)$ & $(17.2)$ & $(20.4)$ \\
\hline & GM_MAP & 0.179 & 0.182 & 0.199 & 0.200 & 0.191 & 0.203 \\
\hline & & & $(2.0)$ & $(11.2)$ & (11.7) & $(6.8)$ & (13.4) \\
\hline & $\mathrm{P} @ 10$ & 0.421 & 0.405 & 0.442 & 0.437 & 0.438 & 0.453 \\
\hline & & & $(-3.8)$ & $(5.0)$ & $(3.8)$ & $(4.1)$ & $(7.4)$ \\
\hline & \#rel_ret & 2887 & 3172 & 3002 & 3143 & 3173 & 3194 \\
\hline & & & $(9.9)$ & $(4.0)$ & $(8.9)$ & $(9.9)$ & (10.6) \\
\hline & $>$ baseline on & 0 & 52 & 56 & 58 & 55 & 60 \\
\hline \multirow[t]{9}{*}{ TREC910 } & MAP & 0.195 & 0.193 & 0.211 & 0.213 & 0.204 & $0.222^{B, k, k l}$ \\
\hline & & & $(-1.1)$ & $(8.0)$ & $(9.0)$ & $(4.7)$ & (13.5) \\
\hline & GM_MAP & 0.081 & 0.056 & 0.087 & 0.081 & 0.063 & 0.073 \\
\hline & & & $(-30.2)$ & $(7.4)$ & $(0.2)$ & $(-22.4)$ & $(-9.2)$ \\
\hline & $\mathrm{P} @ 10$ & 0.307 & 0.293 & 0.329 & 0.336 & 0.313 & 0.319 \\
\hline & & & $(-4.6)$ & $(7.0)$ & $(9.3)$ & $(2.0)$ & $(4.0)$ \\
\hline & \#rel_ret & 3770 & 3987 & 3889 & 3981 & 4021 & 4159 \\
\hline & & & $(5.8)$ & $(3.2)$ & $(5.6)$ & $(6.7)$ & $(10.3)$ \\
\hline & $>$ baseline on & 0 & 44 & 53 & 47 & 51 & 53 \\
\hline
\end{tabular}

Table 4: Improvements obtained using KLWNET on different datasets. The "> baseline on" line shows the \%-age of queries for which each method beats the baseline by $>5 \%$. Superscripts $B, k, r, w, k l$ denote a statistically significant improvement of the proposed method over the baseline (no feedback), KLD, RM3, P-WNET and KLDLCA respectively. For a particular collection, the highest value for any metric is shown in bold.

all collections, KLWNET also emerges as the "safest" or most robust method, in the sense that it is best in terms of the number of queries for which expansion improves retrieval effectiveness. Additionally, KLWNET has the highest recall at 1000 documents for all corpora except TREC5, where KLWNET is second to P-WNET. For the other two measures (GM_MAP, P@10), KLWNET is consistently among the top 3 methods, and differs very little from the best method. Overall, KLWNET seems to perform very well on all measures and for all collections.

Table 5 (similar to Table 3) presents a comparison between KLWNET, RM3, KLD, and $M I I_{m p}$, a method proposed in (Fang, 2008) that combines cooccurrence information with information from Word-

\begin{tabular}{ccccccc}
\hline Dataset & Baseline & $\begin{array}{c}\text { Baseline } \\
(\text { Fang, 2008) }\end{array}$ & KLD & RM3 & KLWNET & $\begin{array}{c}M I I_{m p} \\
\text { (Fang, 2008) }\end{array}$ \\
\hline TREC7 & 0.1891 & 0.1860 & 0.2568 & 0.2025 & 0.2688 & 0.2370 \\
TREC8 & 0.2467 & 0.2500 & 0.2861 & 0.2591 & 0.3013 & 0.2800 \\
\hline
\end{tabular}

Table 5: Comparison between Fang's combination-based method and KLWNET. 


\begin{tabular}{lr}
\hline Methods & \# queries \\
\hline KLWNET $>$ KLDLCA, P-WNET & 40 \\
P-WNET $\geq$ KLWNET $\geq$ KLDLCA & 62 \\
KLDLCA $\geq$ KLWNET $\geq$ P-WNET & 49 \\
\hline
\end{tabular}

Table 6: Query-level comparison of various methods. (For one query, all methods perform equally well; this query is included in the counts in the last two rows of the table.)

\begin{tabular}{lccc}
\hline & P-WNET & KLDLCA & KLWNET \\
\hline $\begin{array}{l}\text { Wt. of original query term } \\
\text { (polygamy) }\end{array}$ & 2.94 & 1.00 & 1.00 \\
Wt. of first exp. term & 1.00 & 0.69 & 0.48 \\
\hline
\end{tabular}

Table 7: Weights of original and expansion terms for query 316

Net. The figures for $M I I_{m p}$ are taken from (Fang, 2008). This method ( $\left.M I I_{m p}\right)$ performs the best among all methods proposed in that paper. The table shows that KLWNET's MAP figures are substantially higher than those for $M I I_{m p}$. KLWNET also outperforms KLD and RM3 on the TREC7 and TREC8 collections.

\section{Discussion}

The results in the preceding subsection confirm our hypothesis that, on average, the combination based method works well. On all collections, KLWNET outperforms both KLDLCA and P-WNET. A closer look at these results (see Table 6) shows that, of the 150 queries in the TREC678 collection, KLWNET does best on 40 queries. For the remaining queries, either KLDLCA or P-WNET performs noticeably poorly. For each of these queries, the combination manages to achieve an intermediate level of performance thanks to the contribution of the superior method. Thus, the MAP obtained using KLWNET lies between the figures corresponding to P-WNET and KLDLCA. Below, we discuss some queries for which this pattern is observed.

P-WNET $\geq$ KLWNET $\geq$ KLDLCA. For about half of these queries, the original query terms appear to be vital. Since P-WNET attaches relatively greater importance to the original query terms (see Equation 8), it does well on these queries. On the other hand, when the query is expanded using KLDLCA, the importance of the original query terms is diluted, resulting in a drop in performance (KLDLCA also makes use of Equations (6) - (8) to compute term weights for the expanded query, but $\beta$ is set to 1 , since this setting yields better overall performance.). Thus, for these queries, even the baseline method yields higher MAP than KLDLCA. Since KLDLCA is the dominant member of the combination method (see Equation 9), KLWNET also ends up performing poorly compared to P-WNET.

Query 316 (Polygamy Polyandry Polygyny) is a typical example. Table 7 compares the weights of original query terms with that of the first (i.e. highest weighted) expansion term. When the query is expanded using P-WNET, the original term polygamy gets a weight of 2.94 (Equation 8), while the first expansion term (widow) gets a weight of 1.00. Under KLDLCA, on the other hand, the first expansion term (children) gets a relatively high weight of 0.6865. For KLWNET (Equation 9), the first expansion term (children) gets a somewhat lower weight of 0.4806. Further, P-WNET includes some some good 
terms like 'widow', 'monogami', and 'polygamist' in the expanded query with significant weights. Among these, only 'widow' is included in the expanded query by KLDLCA, but with a relatively low weight.

KLDLCA $\geq$ KLWNET $\geq$ P-WNET. Query 361 (clothing sweatshops) is an example query of the opposite kind. KLDLCA includes the useful term 'shop', but P-WNET does not. KLWNET therefore includes this term with a lower weight. Both methods add the term 'immigr' (a bad term), but KLDLCA assigns a lower weight to this term compared to P-WNET. The combination method assigns an intermediate weight to this term as well. Naturally, KLWNET yields an Average Precision that lies in between the values corresponding to KLDLCA and P-WNET. Unfortunately, however, no general pattern seems to emerge for this class of queries.

\section{Conclusion}

In this paper, we proposed a new way of using WordNet for Query Expansion. This method outperforms the existing WordNet based methods. It also compares favorably with established QE methods such as KLD and RM3. We also proposed a combination of three QE methods that takes into account different aspects of a candidate expansion term's usefulness. For each candidate expansion term, this method considers its distribution, its statistical association with query terms, and also its semantic relation with the query. The combination of diverse sources of information appears to work well, and yields results that are, on the whole, better than the individual methods involved in the combination.

\section{References}

Abdul-Jaleel, N., Allan, J., Croft, W. B., Diaz, F., Larkey, L. S., Li, X., et al. (2004). UMass at TREC 2004: Novelty and HARD. In TREC.

Amati, G. (2003). Probability Models for Information Retrieval Based on Divergence from Randomness. University of Glasgow.

Amati, G., \& Van Rijsbergen, C. J. (2002, October). Probabilistic models of information retrieval based on measuring the divergence from randomness. ACM Trans. Inf. Syst., 20, 357-389.

Banerjee, S., \& Pedersen, T. (2002). An adapted lesk algorithm for word sense disambiguation using wordnet. In Proceedings of the third international conference on computational linguistics and intelligent text processing (pp. 136-145). Springer-Verlag.

Banerjee, S., \& Pedersen, T. (2003). Extended gloss overlaps as a measure of semantic relatedness. In Proceedings of the 18th International Joint Conference on Artificial Intelligence (pp. 805-810).

Bendersky, M., Metzler, D., \& Croft, W. B. (2011). Parameterized concept weighting in verbose queries. In Proceedings of the 34th international ACM SIGIR Conference on Research and Development in Information Retrieval (pp. 605-614). 
Bendersky, M., Metzler, D., \& Croft, W. B. (2012). Effective query formulation with multiple information sources. In Proceedings of the fifth ACM international conference on Web search and data mining (pp. 443-452)

Bhogal, J., Macfarlane, A., \& Smith, P. (2007, July). A review of ontology based query expansion. Inf. Process. Manage., 43(4), 866-886.

Buckley, C., Singhal, A., \& Mitra, M. (1995). New retrieval approaches using smart: Trec 4. In TREC.

Cao, G., Nie, J.-Y., Gao, J., \& Robertson, S. (2008a). Selecting good expansion terms for pseudorelevance feedback. In Proceedings of the 31st Annual International ACM SIGIR Conference on Research and Development in Information Retrieval (pp. 243-250).

Cao, G., Nie, J.-Y., Gao, J., \& Robertson, S. (2008b). Selecting good expansion terms for pseudorelevance feedback. In Proceedings of the 31st Annual International ACM SIGIR Conference on Research and Development in Information Retrieval (pp. 243-250).

Carpineto, C., Mori, R. de, Romano, G., \& Bigi, B. (2001). An information-theoretic approach to automatic query expansion. ACM Trans. Inf. Syst., 19(1), 1-27.

Carpineto, C., \& Romano, G. (2012, January). A survey of automatic query expansion in information retrieval. ACM Comput. Surv., 44(1), 1:1-1:50.

Carpineto, C., Romano, G., \& Giannini, V. (2002). Improving retrieval feedback with multiple termranking function combination. ACM Trans. Inf. Syst., 20(3), 259-290.

Croft, B., \& Yufeng, J. (1994). An association thesaurus for information retrieval. In RIAO (pp. 146-161).

Cui, H., Wen, J.-R., Nie, J.-Y., \& Ma, W.-Y. (2002). Probabilistic query expansion using query logs. In Proceedings of the 11th international conference on World Wide Web (pp. 325-332). New York, NY, USA: ACM.

Doszkocs, T. E. (1978). An associative interactive dictionary for online bibliographic searching. In Jerusalem conference on information technology (pp. 489-492).

Fang, H. (2008). A re-examination of query expansion using lexical resources. In Proceedings of ACL-08: HLT (pp. 139-147).

Krikon, E., Kurland, O., \& Bendersky, M. (2010, December). Utilizing inter-passage and inter-document similarities for reranking search results. ACM Trans. Inf. Syst., 29(1), 3:1-3:28.

Lavrenko, V., \& Croft, W. B. (2001). Relevance-based language models. In SIGIR (pp. 120-127).

Lesk, M. (1986). Automatic sense disambiguation using machine readable dictionaries: how to tell a pine cone from an ice cream cone. In Proceedings of the 5th Annual International conference on Systems documentation (pp. 24-26).

Li, Y., Luk, W. P. R., Ho, K. S. E., \& Chung, F. L. K. (2007). Improving weak ad-hoc queries using wikipedia asexternal corpus. In Proceedings of the 30th Annual International ACM SIGIR Conference on Research and Development in Information Retrieval (pp. 797-798). New York, NY, 


\section{USA: ACM.}

Liu, S., Liu, F., Yu, C., \& Meng, W. (2004). An effective approach to document retrieval via utilizing wordnet and recognizing phrases. In Proceedings of the 2\%th Annual International ACM SIGIR Conference on Research and Development in Information Retrieval (pp. 266-272).

Lv, Y., \& Zhai, C. (2009). A comparative study of methods for estimating query language models with pseudo feedback. In Proceedings of the 18th ACM Conference on Information and Knowledge Management (pp. 1895-1898).

Lv, Y., \& Zhai, C. (2010). Positional relevance model for pseudo-relevance feedback. In Proceedings of the 33rd international ACM SIGIR Conference on Research and Development in Information Retrieval (pp. 579-586).

Miao, J., Huang, J. X., \& Ye, Z. (2012). Proximity-based rocchio's model for pseudo relevance. In Proceedings of the 35th international ACM SIGIR Conference on Research and Development in Information Retrieval (pp. 535-544).

Pal, D., Mitra, M., \& Datta, K. (2013). Query expansion using term distribution and term association. CoRR, abs/1303.0667.

Pérez-Agüera, J. R., \& Araujo, L. (2008). Comparing and combining methods for automatic query expansion. Research in Computing Science (Proceedings of CICLING 2008, Advances in Natural Language Processing and Applications), 33, 177-188.

Qiu, Y., \& Frei, H.-P. (1993). Concept based query expansion. In Proceedings of the 16th Annual International ACM SIGIR Conference on Research and Development in Information Retrieval (pp. 160-169).

Robertson, S. (2004). Understanding inverse document frequency: On theoretical arguments for idf. Journal of Documentation, 60, 2004.

Robertson, S. E. (1991, January). On term selection for query expansion. J. Doc., 46(4), 359-364.

Salton, G. (Ed.). (1971). The SMART retrieval system - experiments in automatic document processing. Englewood, Cliffs, New Jersey: Prentice Hall.

Voorhees, E. M. (1993). On expanding query vectors with lexically related words. In TREC (pp. 223-232).

Voorhees, E. M. (1994). Query expansion using lexical-semantic relations. In Proceedings of the 17th Annual International ACM SIGIR Conference on Research and Development in Information Retrieval (pp. 61-69).

Xu, J., \& Croft, W. B. (1996). Query expansion using local and global document analysis. In SIGIR (pp. 4-11).

Xu, J., \& Croft, W. B. (2000). Improving the effectiveness of information retrieval with local context analysis. ACM Trans. Inf. Syst., 18(1), 79-112.

Zhang, J., Deng, B., \& Li, X. (2009). Concept based query expansion using wordnet. In Proceedings of 
the 2009 International e-Conference on Advanced Science and Technology (pp. 52-55). 